\title{
ULTRA-HIGH EXTERNAL EFFICIENCY FROM SURFACE TEXTURED THIN-FILM LEDS
}

\author{
I. Schnitzer, E. Yablonovitch, R. Ranganathan, V. Arbet-Engels, and A. Scherer \\ Electrical Engineering Department, UCLA, 405 Hilgard Ave., Los Angeles, CA 90024-1594 \\ RAFAEL, Dept. 23, P.O.Box 2250, Haifa 31021, Israel Caltech, EE Dept., Pasadena, CA 91125
}

Dire need for high external quantum efficiency $\left(\eta_{\mathrm{ext}}\right)$ visible light-emitting-diodes (LED's) is clearly seen as the flat panel display technology is rapidly evolves. However, there is an enormous gap between the theoretical efficiency of LED's and their actual efficiency. It has been known that good quality III-V double heterostructures can have over $90 \%$ internal quantum yields $(\eta)$ for direct band-gap compounds and over $99 \%$ for $\mathrm{AlGaAs} / \mathrm{GaAs} / \mathrm{AlGaAs}$, as we have demonstrated recently. ${ }^{1}$ On the other hand, run-of-the-mill commercial LED's are usually only a few percent efficient (externally).

The reason for this long-standing shortfall is the difficulty for light to escape from high refractive index semiconductor. A mere $2 \%$ of the internally generated light is coupled into free space through the $16^{\circ}$ escape cone, the rest suffering total internal reflection and risking re-absorption. The present commercial state-of-the-art, $15 \%$ external efficiency in red AlGaAs-based LED's, is achieved by growing a thick transparent semiconductor superstrate, and total substrate removal in a particularly clean, low-loss, optical design which can add greatly to the cost. ${ }^{2}$ Applying this design approach to construct greenvellow, AlInGaP-based, LEDs yield a record high $6 \%$ external efficiency for about $50 \%$ internally efficient heterojunction. Improved growth procedure and subsequent removal of the absorbing growth substrate is expected to push their efficiency above $10 \%$. Green and blue emitting diodes, made of either II-VI's or indirect III-V's compounds, suffer from the same poor light extraction efficiency (due to their high refractive index), in addition to their generally low internal quantum yield.

The key to increasing the escape probability, or extraction efficiency, from high refractive index semiconductors is to give the photons multiple opportunities to find the escape cone. This requires angular randomization or scrambling of the light rays. One way to do this is by photon recycling in a very clean, low-loss optical design. In ref. 1 we used the epitaxial liftoff (ELO) technique ${ }^{3}$ to mount thin film heterostructures on high reflectivity surfaces. It was found that while the photon re-cycling can yield an ultrahigh external quantum efficiency $(72 \%)$, it requires impractical high internal quantum efficiency (for visible LED materials) and high self-absorption. A more practical approach, and the one which we have demonstrated recently in GaAs $\mathrm{LEDs}^{4}{ }^{4}$ is the angular randomization by elastic scattering of the photons from a textured semiconductor surface. In nano-textured LED's the randomization efficiency, and therefore the light extraction efficiency, are independent of the material quality, or the active region thickness. Our approach, schematically illustrated in Fig. 1, has two components: i) Separation of thin-film heterojunctions from the growth substrate using the ELO technique, and, ii) Nano-texturing of the thin-film semiconductor interface by natural lithography. ${ }^{5}$

Figure 1: Typical photon trajectory in the thin $(\sim 1 \mu \mathrm{m})$ epi-liftoff heterostructure. Angular randomization is achieved by strong elastic scattering from the textured surface.

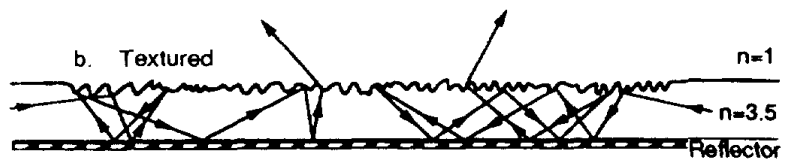

In Ref. 4, 30\% external quantum efficiency has been achieved in GaAs-based LEDs. In this paper we show that an optimized LED design can yield over $50 \%$ efficient GaAs LEDs, and perhaps even more rewarding, close to $50 \%$ efficient thin-film visible LEDs. Moreover, lifting the LED arrays off their growth substrate offer the added advantage of direct integration with Si circuitry for flat panel display or smart slide applications. 
To estimate an upper limit to the external quantum efficiency of thin-film LEDs we invoke a statistical approach ${ }^{6}$ which describes the ergodic photon trajectories within the thin film semiconductor. We first note that the outflow from the trapped ergodic photon gas, through the top (textured) surface in Fig. 1 is $\pi B T / n^{2}$ per unit arca, where $B$ is the brightness of the trapped photon gas, $n$ is the average refractive index of the film, and $T$ is the angle averaged transmission (over the escape cone) through the semiconductor/air interface. This favorable escape rate competes with the dissipative loss rates due to absorption at the rear reflector surface, $\pi B(1-R)$, and due to non-recycled bulk absorption (predominately free carrier absorption) given by $4 \pi B \Sigma_{a_{j}} d_{j}$, where $R$ is the angle averaged bottom interface reflectivity, and $\alpha_{j}$ and $d_{j}$ are the absorptivity and thickness of the film's $j$ th layer, respectively. Finally, the light extraction efficiency from the ergodic photon gas is given by the ratio between the outflow through the escape cone (top surface), divided by the photon inflow which is balanced by the total photon outflow through both the desirable and dissipative loss channels above. Therefore,

$$
\eta_{\mathrm{ext}}=\eta\left(1+\operatorname{Ln}^{2} / \mathrm{T}\right)^{-1} \sim \eta /(1+18 \mathrm{~L})
$$

where $L=(1-R)+\Sigma_{a_{j}} d_{j}$ is the double pass parasitic absorption and $n \sim 3.5$ was assumed for the final approximation. Note that the expression above is in fact an underestimated limit for the maximum external efficiency in that we ignore photon recycling (by minimizing the active layer absorptivity).

The advantage of the textured thin-film geometry is now evident: if the parasitic losses are kept below $5 \%$ (reasonable requirement), the extraction efficiency $\left(\eta_{\text {exr }} / \eta\right)$ can be over $50 \%$. Currently, the internal quantum efficiency of AlInGaP-based heterojunctions is about $50 \%$ which suggests the feasibility of $25 \%$ efficient visible LED arrays, a four-fold improvement of state-of-the-art visible LEDs. The same applies to II-VI blue green light emitters, once separated from their absorbing substrate.

Figure 2: The surface nano-texturing lies in the heart of our thin-film geometry. In Natural Lithography. Polystyrene spheres, $0.2 \mu \mathrm{m}$ diameter, coat the surface of the LED surface in a randomly close-packed array using the surface forces between the charged spheres and the semiconductor. The spheres then act as an etch mask for a $\mathrm{Cl}_{2}$ assisted $\mathrm{Xe}^{+}$ion beam etching, about $0.17 \mu \mathrm{m}$ deep. The spheres can be easily washed away to leave a clean high refractive index textured surface.

The fact that Natural Lithography is applied to a large thin-film LED dense array, mounted on any smooth substrate, may become extremely valuable in flat panel display technology.

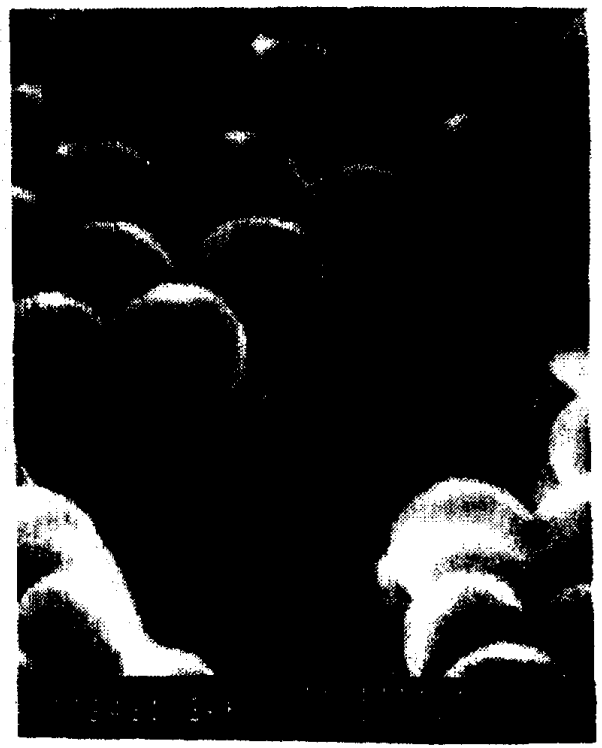

1. 1. Schnitzer, E. Yablonovitch, C. Caneau, and T. J. Gmitter, Appl. Phys. Lett. 62, 131 (1993).

2. R. M. Fletcher, C. Kuo, T. D. Osentowski, J. G. Yu, and V. Robbins, Hewlett-Packard Journal pp. 6-14, August 1993 issue and references therein.

3. E. Yablonovitch, T. J. Gmitter, J. P. Harbison, and R. Bhat, Appl. Phys. Lett. 51, 2222 (1987).

4. I. Schnitzer, E. Yablonovitch, C. Caneau, T. J. Gmitter, A. Scherer, Appl. Phys. Lett. 63, 2174 (1993).

5. H. W. Deckman and J. H. Dunsmuir, A ppl. Phys. Lett. 41, 377 (1982).

6. E. Yablonovitch, J. Opt. Soc. Am. 72, 899 (1982). 